\title{
A comparison of transcutaneous bilirubinometers: SpectRx BiliCheck versus Minolta AirShields
}

\author{
C M Wong, P J E van Dijk, I A Laing
}

Arch Dis Child Fetal Neonatal Ed 2002;87:F 137-F 140

See end of article for authors' affiliations ....................

Correspondence to: Dr Wong, Neonatal Unit (Ward 110), Royal Infirmary of Edinburgh at Little France, 51 Little France Crescent, Edinburgh EH16 4SA, Scotland, UK; mae.wong@luht.scot.nhs.uk

Accepted 22 March 2002
Background: Two devices are available for making transcutaneous estimates of serum bilirubin (SBR): the Minolta AirShields JM102 and the new SpectRx BiliCheck.

Objectives: (a) To measure how well the readings produced by these devices agree with SBR measured in the laboratory; (b) to estimate for each device, the proportion of infants with clinical jaundice who would require blood sampling if the device was used as a screening tool to detect infants with $\mathrm{SBR} \geqslant 250 \mu \mathrm{mol} / \mathrm{l}$.

Design: Prospective cohort study of jaundiced infants who required SBR at $\leqslant 20$ days of postnatal age. Those who had received phototherapy or exchange transfusion were excluded.

Setting: Tertiary neonatal service in South-East Scotland.

Interventions: Within 30 minutes of SBR sampling, transcutaneous bilirubinometry was performed using one Minolta and two SpectRx devices (designated A and B).

Results: Sixty four neonates were enrolled, 19 of which were preterm (31-35 weeks). The 95\% confidence intervals of a device reading corresponding to SBR were $\pm 66.7, \pm 67.9$, and $\pm 66.4 \mu \mathrm{mol} / \mathrm{l}$ respectively. Using the devices to identify all SBR $\geqslant 250 \mu \mathrm{mol} / \mathrm{I}$ would reduce SBR sampling by $23 \%$, $16 \%$, and $20 \%$ respectively.

Conclusions: Given that SBR levels range from 50 to $400 \mu \mathrm{mol} / \mathrm{l}$ in jaundiced infants, the $95 \%$ confidence intervals of the devices are wide at $\pm 67 \mu \mathrm{mol} / \mathrm{l}$. The SpectRx can be used as a screening tool for hyperbilirubinaemia but there is no advantage in using it over the Minolta.
N eonatal hyperbilirubinaemia is a common phenomenon but relatively few affected infants require intervention. Whatever the cause, total serum bilirubin (SBR) levels above defined thresholds warrant treatment to prevent the development of kernicterus.

The clinical evaluation of hyperbilirubinaemia involves visual estimation of the yellowness of skin, known as jaundice. However, quantification of SBR based on visual assessment of the depth of jaundice is subjective and inaccurate, and confounded by skin colour and haemoglobin. ${ }^{1}$ Various methods have therefore been developed to aid non-invasive diagnosis of hyperbilirubinaemia and approximation of SBR levels. They include cephalocaudal staging of jaundice $^{23}$ and transcutaneous reference devices. However, because of limited accuracy, these transcutaneous devices have only been useful as screening rather than diagnostic tools for determining hyperbilirubinaemia. They include the Ingram Icterometer ${ }^{2}{ }^{4}$ Chromatics Colormate III, ${ }^{5}$ and Minolta AirShields Jaundice Meter. ${ }^{46}$ A new device, the SpectRx BiliCheck, is claimed to have high accuracy and reproducibility across different ethnic groups and gestational ages, although the authors do state that further assessment of efficacy is needed for SBR $\geqslant 256 \mu \mathrm{mol} / \mathrm{l}^{7}$ It has also been suggested that the SpectRx device is accurate enough to be a substitute for SBR sampling. ${ }^{8}$

Two devices are available for making transcutaneous estimates of SBR, the Minolta AirShields JM102 and the new SpectRx BiliCheck. The aims of our study were to $(a)$ measure how well the readings produced by these devices agree with SBR measured in the laboratory, and $(b)$ estimate for each device the proportion of infants with clinical jaundice who would require blood sampling if the device was used as a screening tool to detect infants with SBR $\geqslant 250 \mu \mathrm{mol} / \mathrm{l}$. This threshold was used to define significant hyperbilirubinaemia, as most infants affected would require either treatment or further investigation of their hyperbilirubinaemia, and hence would be need to be identified. ${ }^{9}$ This is with the exception of clinical jaundice in the first 24 hours, which warrants investigation whatever the SBR level. ${ }^{9}$ We also assessed the intradevice precision of the Minolta and SpectRx devices. Interdevice precision between two SpectRx devices was evaluated.

\section{METHODS}

Neonates at the Simpson Memorial Maternity Pavilion, Edinburgh, who required blood sampling for SBR were enrolled in the study with parental consent. All neonates were clinically jaundiced but otherwise well. Three bilirubinometers (see below) were applied to the infant's forehead within 30 minutes of SBR sampling by venepuncture or heel lance. All transcutaneous bilirubinometry (TcB) measurements were performed by one author (PvD) with the infant lying supine. The forehead was not exposed to direct sunlight and care was taken to avoid skin areas that were bruised, excessively hairy, or hypermelanotic. Gestation, birth weight, postnatal age, and ethnicity were recorded. Infants who had received phototherapy or exchange transfusion were excluded.

SBR samples were sent immediately for analysis by an automated Hitachi 911 multichannel analyser. The method used involves oxidation of bilirubin to biliverdin by potassium ferricyanide in the presence of caffeine. ${ }^{10}$ This technique is reliable even in the presence of haemolysis up to $5.0 \mathrm{~g} / \mathrm{l}$ and is therefore particularly suitable for neonatal samples. Interbatch quality control coefficients of variation are $3.0 \%$ at 53 $\mu \mathrm{mol} / \mathrm{l}, 1.4 \%$ at $124 \mu \mathrm{mol} / \mathrm{l}$, and $1.6 \%$ at $231 \mu \mathrm{mol} / \mathrm{l}$. The laboratory participates in the United Kingdom External Quality Assessment Scheme, and shows a mean $+2.4 \%$ bias for SBR analysis.

Abbreviations: $S B R$, serum bilirubin; $T c B$, transcutaneous bilirubinometry; HPLC, high performance liquid chromatography 
Table 1 Details of patients enrolled in the study

\begin{tabular}{|c|c|c|c|}
\hline & Mean & SD & Range \\
\hline \multicolumn{4}{|l|}{ All infants $(n=64)$} \\
\hline Gestation (weeks) & 37.4 & 3.0 & $31-42$ \\
\hline Birth weight (g) & 2920.8 & 755.5 & $3000-4610$ \\
\hline SBR ( $\mu \mathrm{mol} / \mathrm{l})$ & 207.3 & 68.8 & $50-349$ \\
\hline Postnatal age (days) & 4.6 & 3.4 & $1-17$ \\
\hline \multicolumn{4}{|l|}{ Term infants $(n=45)$} \\
\hline Gestation (weeks) & 39.1 & 1.4 & $37-42$ \\
\hline Birth weight (g) & 3258.9 & 605.4 & $1750-4610$ \\
\hline 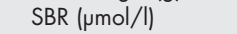 & 212.1 & 72.5 & $50-349$ \\
\hline Postnatal age (days) & 3.6 & 2.5 & $1-14$ \\
\hline \multicolumn{4}{|l|}{ Preterm infants $(n=19)$} \\
\hline Gestation (weeks) & 33.4 & 1.2 & $31-35$ \\
\hline Birth weight (g) & 2120.0 & 373.1 & $1610-3120$ \\
\hline SBR ( $\mu \mathrm{mol} / \mathrm{l})$ & 195.9 & 59.4 & $85-307$ \\
\hline Postnatal age (days) & 7.0 & 4.0 & $2-17$ \\
\hline
\end{tabular}

SBR, Serum bilirubin.

\section{Bilirubinometer specifications and method of use}

The devices compared were two SpectRx BiliCheck (SpectRx, USA), designated A and B, and one Minolta AirShields JM102 (now Hill-Rom AirShields International). Both systems rely on spectrophotometric methods.

Detailed information on the SpectRx device has been given elsewhere. ${ }^{7}$ The SpectRx instruction manual and website claim the device to be accurate at gestational age 27-42 weeks, postnatal age 0-20 days, and weight $950-5200 \mathrm{~g}$. It is also claimed to be accurate regardless of skin colour by compensating for the confounding effects of dermal maturity, melanin, and haemoglobin on spectral reflectance. The SpectRx uses disposable probe tips which require calibration before each measurement. The tip is then applied with light pressure to the skin. The SpectRx device is designed to be triggered for five consecutive spectral analyses before it provides one displayed averaged reading in either $\mu \mathrm{mol} / \mathrm{l}$ or $\mathrm{mg} / \mathrm{dl}$. In other words, it has an internal averaging system in an attempt to improve accuracy. For the study, three of these readings were obtained per device to determine intradevice precision. The average of these readings was used as the operative result in all comparisons with the Minolta, and for calculating interdevice precision.

The Minolta AirShields also measures the spectral reflectance of bilirubin but does not claim to correct for confounding effects. Detail on operation principles is described elsewhere. ${ }^{6}$ It has been extensively studied and validated for use as a screening tool for hyperbilirubinaemia in term infants. ${ }^{41-13}$ There have also been reports in the literature on its use in ethnic minorities with various skin colouring. ${ }^{112}{ }^{14}{ }^{15}$ The Minolta device does not use disposable parts or require repeated calibration. The probe tip is applied to the skin with increasing pressure until a flash is activated and a reading obtained. Readings are produced as a numeric index. The Minolta was used according to the protocol of the study centre, recording three consecutive readings, and choosing the maximum value for clinical decision making.

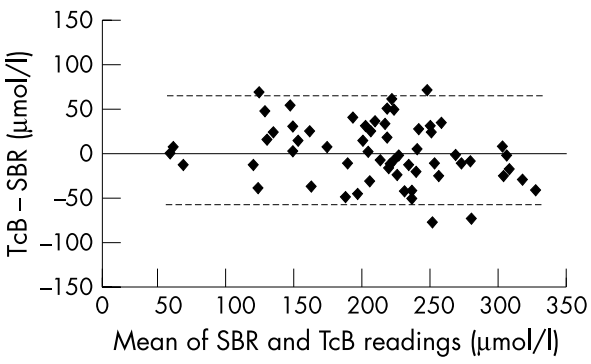

Figure 1 Bland-Altman plots for the Minolta device. The dotted line represents $95 \%$ confidence intervals of the difference, \pm 66.7 $\mu \mathrm{mol} / \mathrm{l}$. SBR, Serum bilirubin; TcB, transcutaneous bilirubinometry.

\section{Data and statistical analysis}

For statistical accuracy, each patient was assessed only once. All statistical calculations were performed using Microsoft Excel 97. Pearson correlation coefficients were calculated using original Minolta index readings. For measurement of agreement by the Bland-Altman method, ${ }^{16}$ Minolta index readings were transformed into $\mu \mathrm{mol} / \mathrm{l}$ using linear regression of SBR on TCB readings. The transformed values were used for calculation of the differences between SBR and TcB values, the mean difference and $95 \%$ confidence intervals of the difference.

\section{RESULTS}

From June to September 2000, 64 neonates were enrolled, 19 of whom were preterm (31-35 weeks). Table 1 gives patient details. All were less than 20 days old. Six infants were non-white. None had a haemoglobinopathy. SBR levels ranged from 50 to $349 \mu \mathrm{mol} / \mathrm{l}$.

\section{Agreement between SpectRx and Minolta devices and SBR}

Table 2 shows agreement results for TcB readings against SBR. The $95 \%$ confidence intervals of a device reading corresponding to SBR were $\pm 66.7, \pm 67.9$, and $\pm 66.4 \mu \mathrm{mol} / \mathrm{l}$ for the Minolta, SpectRx A, and SpectRx B respectively. The BlandAltman scatterplots for the devices show a tendency for both Minolta and SpectRx devices to underestimate SBR at higher levels (figs 1-3).

For preterm infants, the Minolta device showed a tendency to overestimate SBR but the device has not been validated for such use. A good correlation with SBR was seen in non-white infants for the Minolta, SpectRx A, and SpectRx B $(r=0.94$, 0.95 , and 0.99 respectively), but because of small numbers $(\mathrm{n}=6)$, it was deemed inappropriate to calculate statistical agreement.

Accuracy can be determined by agreement, but for practical purposes (such as defining thresholds for performing SBR), the maximum differences between device readings and SBR should be considered. Consequently 100\% of infants with hyperbilirubinaemia requiring treatment would be identified. Table 2 also displays the maximum range of differences between devices and SBR. For example, to identify $100 \%$ of

Table 2 Mean and maximum differences, and 95\% confidence intervals of the differences for respective devices

\begin{tabular}{|c|c|c|c|c|c|c|c|c|c|c|}
\hline \multirow[b]{2}{*}{ Patients } & \multirow[b]{2}{*}{ No } & \multicolumn{3}{|c|}{ Minolta } & \multicolumn{3}{|c|}{ SpectRx A } & \multicolumn{3}{|c|}{ SpectRx B } \\
\hline & & $\mathrm{m} \Delta$ & $95 \% \mathrm{Cl}$ & $\operatorname{Max} \Delta$ & $\mathrm{m} \Delta$ & $95 \% \mathrm{Cl}$ & $\operatorname{Max} \Delta$ & $\mathrm{m} \Delta$ & $95 \% \mathrm{Cl}$ & $\operatorname{Max} \Delta$ \\
\hline All & 64 & 0.0 & \pm 66.7 & -79 to +69 & -4.0 & \pm 67.9 & -88 to +88 & -8.6 & \pm 66.4 & -101 to +67 \\
\hline Term & 45 & -9.6 & \pm 65.1 & & -5.5 & \pm 67.2 & & -12.8 & \pm 62.9 & \\
\hline Preterm & 19 & 22.7 & \pm 46.0 & & -0.5 & \pm 71.1 & & 1.3 & \pm 72.0 & \\
\hline
\end{tabular}

$\mathrm{m} \Delta(\mu \mathrm{mol} / \mathrm{I})=$ mean difference $($ device$-\mathrm{SBR}) ; 95 \% \mathrm{Cl}(\mu \mathrm{mol} / \mathrm{l})=95 \%$ confidence intervals of the difference; Max $\Delta(\mu \mathrm{mol} / \mathrm{I})=$ maximum range of difference (device-SBR); SBR, serum bilirubin. 


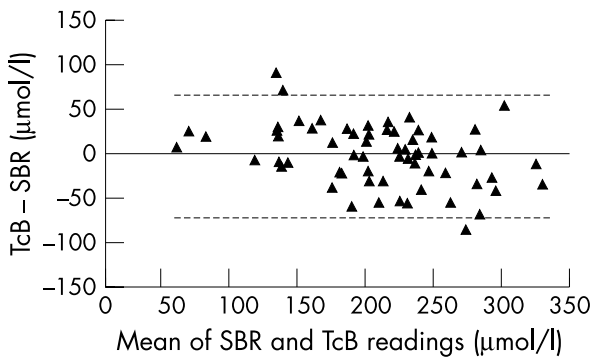

Figure 2 Bland-Altman plots for the SpectRx A device. The dotted line represents $95 \%$ confidence intervals of the difference, \pm 67.9 umol/I. SBR, Serum bilirubin; TcB, transcutaneous bilirubinometry.

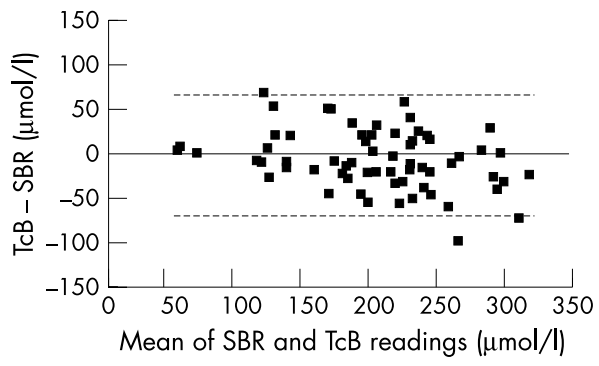

Figure 3 Bland-Altman plots for the SpectRx B device. The dotted line represents $95 \%$ confidence intervals of the difference, \pm 66.4 $\mu \mathrm{mol} / \mathrm{l}$. SBR, Serum bilirubin; TcB, transcutaneous bilirubinometry.

infants with $\mathrm{SBR} \geqslant 250 \mu \mathrm{mol} / \mathrm{l}$, blood samples need to be obtained from all infants whose Minolta reading is $\geqslant 170$ $\mu \mathrm{mol} / \mathrm{l}$ (index reading 15.5 ) or whose SpectRx reading is $\geqslant 150 \mu \mathrm{mol} / \mathrm{l}$.

\section{Positive predictive value and number of SBR samples required to identify all $S B R \geqslant 250 \mu \mathrm{mol} / \mathrm{I}$}

When the threshold for each device is defined to achieve $100 \%$ sensitivity for SBR $\geqslant 250 \mu \mathrm{mol} / \mathrm{l}$, the positive predictive values are similar (table 3 ). Using the devices to screen for this threshold would reduce SBR sampling by $23 \%$ for the Minolta device, 16\% for SpectRx A, and 20\% for SpectRx B. Thus the SpectRx does not result in fewer SBR samples being taken to identify all infants with significant hyperbilirubinaemia.

\section{Intradevice precision}

The median coefficients of variation for the Minolta, SpectRx A, and SpectRx B were $1.49 \%, 8.94 \%$, and $8.51 \%$ respectively.
Maximum coefficients of variation were $6.74 \%, 18.67 \%$, and $27.64 \%$ respectively.

\section{Interdevice precision}

The $95 \%$ confidence interval of difference in readings for the two SpectRx devices was $\pm 37.4 \mu \mathrm{mol} / \mathrm{l}$.

\section{DISCUSSION}

This study is the first to compare the performance of the new SpectRx BiliCheck with the Minolta AirShields. Given that SBR levels range from 50 to $400 \mu \mathrm{mol} / \mathrm{l}$ in jaundiced infants, both instruments have wide $95 \%$ confidence intervals at \pm 67 $\mu \mathrm{mol} / \mathrm{l}$. A TcB reading that is different from SBR by up to 100 $\mu \mathrm{mol} / \mathrm{l}$ (the maximum difference in this study) can have implications for starting phototherapy or exchange transfusion. ${ }^{9}$ Such a difference could possibly also have an effect on whether an infant develops kernicterus or not. These devices are useful as screening tools for hyperbilirubinaemia, but not as replacements for measuring SBR.

\section{Comparison of SpectRx and Minolta devices}

Our study has shown that, as a screening tool, there is no advantage in using the SpectRx device over the Minolta. Although the SpectRx provides readings in $\mu \mathrm{mol} / \mathrm{l}$ for easier interpretation, and claims to be accurate regardless of skin colour, we have found that it is neither more accurate than the Minolta device nor does it reduce the number of samples needed to identify significant hyperbilirubinaemia. Agreement is a better measure of accuracy than correlation, and the agreement between the device readings and SBR is poor.

Positive predictive values are acknowledged to vary with disease prevalence, in this case the number of jaundiced infants deemed to require SBR sampling. Hence our positive predictive values should not be extrapolated to a different population of infants, but nevertheless provide useful data for cross comparisons between the devices. The positive predictive values of the devices were similar for all three devices.

Clinical usefulness of the devices as screening tools was assessed by the reduction in SBR sampling while ensuring that all samples with SBR $\geqslant 250 \mu \mathrm{mol} / \mathrm{l}$ were identified. Thus for the Minolta, SpectRx A, and SpectRx B devices respectively, $15(23 \%), 10(16 \%)$, and 13 (20\%) SBR samples could have been avoided. The Minolta, in fact, performed slightly better with respect to reduction of SBR sampling than the SpectRx devices. Again, these data are valuable primarily for comparing the devices with each other.

Whichever screening tool is used, the Bland-Altman scatterplots show a tendency for the devices to underestimate

\begin{tabular}{|c|c|c|c|c|}
\hline & $\mathrm{SBR} \geqslant 250 \mu \mathrm{mol} / / \mathrm{l}$ & $\mathrm{SBR}<250 \mu \mathrm{mol} / \mathrm{l}$ & Totals & $\begin{array}{l}\text { Positive predictive } \\
\text { value }\end{array}$ \\
\hline \multicolumn{5}{|l|}{ Minolta } \\
\hline TcB reading $\geqslant 170 \mu \mathrm{mol} / / \mathrm{l}$ & 17 & 32 & 49 & \multirow[t]{3}{*}{34.7} \\
\hline TCB reading $<170 \mu \mathrm{mol} / \mathrm{l}$ & 0 & 15 & 15 & \\
\hline Totals & 17 & 47 & 64 & \\
\hline \multicolumn{5}{|l|}{ SpectRx A } \\
\hline TcB reading $\geqslant 150 \mu \mathrm{mol} / / \mathrm{l}$ & 17 & 37 & 54 & \multirow[t]{4}{*}{31.5} \\
\hline TcB reading $<150 \mu \mathrm{mol} / \mathrm{l}$ & 0 & 10 & 10 & \\
\hline \multirow[t]{2}{*}{ Totals } & & & \multirow[t]{2}{*}{64} & \\
\hline & 100.0 (sensitivity) & 21.3 (specificity) & & \\
\hline \multicolumn{5}{|l|}{ SpectRx B } \\
\hline TcB reading $\geqslant 150 \mu \mathrm{mol} / / \mathrm{l}$ & 17 & 34 & 51 & \multirow[t]{4}{*}{33.3} \\
\hline$T c B$ reading $<150 \mu \mathrm{mol} / / \mathrm{l}$ & 0 & 13 & 13 & \\
\hline Totals & 17 & 47 & 64 & \\
\hline & 100.0 (sensitivity) & 27.7 (specificity) & & \\
\hline
\end{tabular}

SBR, Serum bilirubin; TcB, transcutaneous bilirubinometry. 
SBR at higher levels. This implies that the devices are less accurate when the risk for kernicterus is higher.

\section{Accuracy of SpectRx device}

Although the aim of the study was to compare the SpectRx with the Minolta, we simultaneously evaluated accuracy for validation purposes. At that time, no published data on the SpectRx device were yet available in the medical literature. Since then, three papers have been published on the accuracy of the SpectRx device. ${ }^{7817}$ The correlation coefficient for the SpectRx in this study $(r=0.87)$ is comparable to those obtained by Bhutani et $a l^{7}(r=0.91)$, Rubaltelli et $a l^{8}(r=0.89)$ and Knupfer et $a l^{17}(r=0.73)$. The $95 \%$ confidence intervals of the difference are also similar at $\pm 67 \mu \mathrm{mol} / \mathrm{l}$ in this study, \pm 55 $\mu \mathrm{mol} / \mathrm{l}$ in Bhutani et al, ${ }^{7}$ and $\pm 76 \mu \mathrm{mol} / \mathrm{l}$ in Rubaltelli et al. ${ }^{8}$ Our data are thus analogous to studies carried out elsewhere and add to the literature about the validity of the SpectRx device.

\section{TcB as a replacement for SBR sampling}

Perhaps one limitation of this study is that SBR was analysed by a clinical laboratory method rather than the ideal method of high performance liquid chromatography (HPLC). It has been argued that, when compared with HPLC, some clinical laboratory analyses of SBR use automated methods that are subject to errors as wide as those obtained with TcB. ${ }^{8}$ Our study used the Hitachi 911 multichannel analyser with the potassium ferricyanide method, which has been shown to be an accurate and precise assay for total SBR..$^{10}$ Furthermore, our laboratory participates in rigorous internal and external quality control procedures. Each institution should be aware of the accuracy of their local clinical laboratory method for measuring SBR before deciding whether transcutaneous devices are suitable replacements.

Rubaltelli et al $^{8}$ propose that transcutaneous measurements could be used to replace invasive SBR sampling altogether. They reported correlation coefficients for SpectRx compared with HPLC $(r=0.89)$, and laboratory SBR with HPLC $(r=0.93)$. The corresponding $95 \%$ confidence intervals of the difference were $\pm 76 \mu \mathrm{mol} / \mathrm{l}$ and $\pm 63 \mu \mathrm{mol} / \mathrm{l}$ (non-significant at $\mathrm{p}=0.53$ ). Despite not reaching statistical significance, the 95\% confidence intervals were superior for laboratory SBR. The authors concluded that the SpectRx performed as well as laboratory SBR. However, the overall accuracy of their laboratory SBR results was compromised by the use of nine different automated laboratory methods. It is known that certain methods perform more accurately than others, ${ }^{18}$ but no information was provided about the individual performance of each of the nine methods.

In addition, no studies have addressed the question of whether skin or serum levels of bilirubin more accurately reflect its deposition in the central nervous system. Some suggest that transcutaneous readings are more representative of tissue deposition of bilirubin, ${ }^{19}{ }^{20}$ whereas others propose that circulating serum bilirubin is more available to move across the blood-brain barrier. ${ }^{8}$ Factors that affect blood-tissue bilirubin distribution include exchange transfusion, phototherapy, albumin levels, $\mathrm{pH}$, and gestational and postnatal age. This debate on the use of TcB as a replacement for SBR sampling would be ideally resolved by conducting a randomised controlled trial of the two diagnostic methods with long term outcome measurements and cost-benefit analyses. This would require a large, multicentre setting with years of multidisciplinary follow up, and the cost would be prohibitive. The current evidence also shows that the transcutaneous methods at present available are not accurate enough to proceed with such a trial.

\section{Summary}

The purpose of measuring bilirubin levels, by whatever method, is to identify infants who require treatment. The inaccuracy of existing transcutaneous bilirubinometers means that they should only be used as screening tools for SBR sampling. The newer SpectRx BiliCheck is no more accurate than the Minolta AirShields, and is more complicated to use.

\section{Authors' affiliations}

C M Wong, P J E van Diik, I A Laing, Simpson Memorial Maternity Pavilion, Lauriston Place, Edinburgh EH3 9YW, Scotland, UK

\section{REFERENCES}

1 Moyer VA, Ahn C, Sneed S. Accuracy of clinical judgment in neonatal jaundice. Arch Pediatr Adolesc Med 2000;154:391-4.

2 Madlon-Kay DJ. Recognition of the presence and severity of newborn jaundice by parents, nurses, physicians, and icterometer. Pediatrics 1997; 100:E3.

3 Knudsen A. The cephalocaudal progression of jaundice in newborns in relation to the transfer of bilirubin from plasma to skin. Early Hum Dev 1990;22:23-8

4 Schumacher RE, Thornbery JM, Gutcher GR. Transcutaneous bilirubinometry: a comparison of old and new methods. Pediatrics 1985:76:10-14.

5 Tayaba R, Gribetz D, Gribetz I, et al. Noninvasive estimation of serum bilirubin. Pediatrics 1998;102:E28

6 Yamanouchi I, Yamauchi Y, Igarashi I. Transcutaneous bilirubinometry: preliminary studies of noninvasive transcutaneous bilirubin meter in the Okayama National Hospital. Pediatrics 1980;65:195-202.

7 Bhutani VK, Gourley GR, Adler S, et al. Noninvasive measurement of total serum bilirubin in a multiracial predischarge newborn population to assess the risk of severe hyperbilirubinemia. Pediatrics 2000;106:E17.

8 Rubaltelli FF, Gourley GR, Loskamp N, et al. Transcutaneous bilirubin measurement: a multicenter evaluation of a new device. Pediatrics 2001;107:1264-71.

9 Practice parameter: management of hyperbilirubinemia in the healthy term newborn. American Academy of Pediatrics. Provisional Committee for Quality Improvement and Subcommittee on Hyperbilirubinemia. Pediatrics 1994;94:558-65.

10 O'Leary N, Pembroke A, Duggan PF. A robust procedure for the automated measurement of total serum bilirubin using potassium ferricyanide. Ann Clin Biochem 1993;30:175-9

11 Hegyi T, Hiatt IM, Indyk L. Transcutaneous bilirubinometry. I. Correlations in term infants. J Pediatr 1981;98:454-7.

12 Yamauchi Y, Yamanouchi I. Transcutaneous bilirubinometry. Evaluation of accuracy and reliability in a large population. Acta Paediatr Scand 1988;77:791-5

13 Briscoe L, Clark S, Yoxall B. Can transcutaneous bilirubinometry reduce the need for blood tests in jaundiced full term babies? Arch Dis Child Fetal Neonatal Ed 2002;86:190-2

14 Tan KL, Mylvaganam A. Transcutaneous bilirubinometry in preterm very low birthweight infants. Acta Paediatr Scand 1988;77:796-801.

15 Tan KL, Chia HP, Koh BC. Transcutaneous bilirubinometry in Chinese, Malay and Indian infants. Acta Paediatr 1996;85:986-90.

16 Bland JM, Altman DG. Statistical methods for assessing agreement between two methods of clinical measurement. Lancet 1986;1:307-10.

17 Knupfer M, Pulzer F, Braun L, et al. Transcutaneous bilirubinometry in preterm infants. Acta Paediatr 2001;90:899-903.

18 Gourley GR, Bhutani VK, Johnson L, et al. Measurement of serum bilirubin in newborn infants: common clinical laboratory methods versus high performance liquid chromatography (HPLC) [abstract]. Pediatr Res 1999;45:283A

19 Knudsen A, Brodersen R. Skin colour and bilirubin in neonates. Arch Dis Child 1989:64:605-9.

20 Knudsen A, Ebbesen $\mathrm{F}$, Hansen $\mathrm{H}$, et al . The increase of yellow skin colour beyond that of serum bilirubin: a proposed indicator of risk for bilirubin encephalopathy in the newborn. Acta Paediatr Jpn 1993;35:418-22. 